\title{
An Integrated Molecular Map of Yellow Passion Fruit Based on Simultaneous Maximum-likelihood Estimation of Linkage and Linkage Phases
}

\author{
Eder J. Oliveira ${ }^{1}$, Maria Lucia C. Vieira², Antonio Augusto F. Garcia, Carla F. Munhoz, \\ Gabriel R.A. Margarido, Luciano Consoli, Frederico P. Matta, and Michel C. Moraes \\ Departamento de Genética, Escola Superior de Agricultura “Luiz de Queiroz”, Universidade de São \\ Paulo, Piracicaba, P.O. Box 83, 13400-970, Brazil
}

\author{
Maria I. Zucchi \\ Centro de Pesquisa e Desenvolvimento em Recursos Genéticos Vegetais, Instituto Agronômico, \\ Campinas, P.O. Box 28, 13012-970, Brazil \\ Maria Helena P. Fungaro \\ Departamento de Biologia Geral, Centro de Ciências Biológicas, Universidade Estadual de Londrina, \\ Londrina, P.O. Box 6001, 86051-990, Brazil
}

\begin{abstract}
Additional Index words. Passiflora edulis f. flavicarpa, microsatellites, AFLP, linkage map integration, outcrossing species, multipoint distances

Abstract. The development of genetic maps for auto-incompatible species, such as the yellow passion fruit (Passiflora edulis Sims f. flavicarpa Deg.) is restricted due to the unfeasibility of obtaining traditional mapping populations based on inbred lines. For this reason, yellow passion fruit linkage maps were generally constructed using a strategy known as two-way pseudo-testcross, based on monoparental dominant markers segregating in a 1:1 fashion. Due to the lack of information from these markers in one of the parents, two individual (parental) maps were obtained. However, integration of these maps is essential, and biparental markers can be used for such an operation. The objective of our study was to construct an integrated molecular map for a full-sib population of yellow passion fruit combining different loci configuration generated from amplified fragment length polymorphisms (AFLPs) and microsatellite markers and using a novel approach based on simultaneous maximum-likelihood estimation of linkage and linkage phases, specially designed for outcrossing species. Of the total number of loci, $\approx 76 \%, 21 \%, 0.7 \%$, and $2.3 \%$ did segregate in 1:1, 3:1, 1:2:1, and 1:1:1:1 ratios, respectively. Ten linkage groups (LGs) were established with a logarithm of the odds (LOD) score $\geq 5.0$ assuming a recombination fraction $\leq \mathbf{0 . 3 5}$. On average, 24 markers were assigned per LG, representing a total map length of $1687 \mathrm{cM}$, with a marker density of $6.9 \mathrm{cM}$. No markers were placed as accessories on the map as was done with previously constructed individual maps.
\end{abstract}

South America is considered the main center of genetic diversity of the genus Passiflora L., where $\approx 450$ species are found from sea level to the Andes Mountains. Although few species are grown commercially for their edible and aromatic fruits, Passiflora edulis is cultivated in several tropical and subtropical areas worldwide. Its yellow form, $P$. edulis $\mathrm{f}$. flavicarpa, is planted in almost all of the commercial orchards in Brazil, where the fruit is consumed in natura or processed industrially to produce juice. The juice is manufactured on a small scale for export to EU countries. Passion vines are susceptible to the bacterium Xanthomonas axonopodis Vau-

\footnotetext{
Received for publication 26 Mar. 2007. Accepted for publication 5 Aug. 2007. We are grateful to the Brazilian Institutions: Fundação de Amparo à Pesquisa do Estado de São Paulo (grant number 03/06074-4), Conselho Nacional de Desenvolvimento Científico e Tecnológico (CNPq), and Coordenação de Aperfeiçoamento de Pessoal de Nível Superior (CAPES). The latter two are acknowledged for fellowships given to our students, post-doctoral researcher (L.C.), and researchers (A.A.F.G., M.H.P.F., and M.L.C.V.).

We thank Christopher P. Burden for his kind contribution in the proofreading of the manuscript.

${ }^{1}$ Current address: Embrapa Mandioca e Fruticultura Tropical, Cruz das Almas, 44380-000, Brazil.

${ }^{2}$ Corresponding author. E-mail: mlcvieir@esalq.usp.br.
}

terin et al., 1995 pv. passiflorae (Pereira) Gonçalves and Rosato, which in recent years has resulted in great losses to the juice industry and fruit producers, mostly in southeastern Brazil (Vieira and Carneiro, 2004, and references therein).

The wide genetic variability, known to exist within $P$. edulis f. flavicarpa, can be exploited in natural and breeding populations and is of interest to localize genomic regions that might affect variation for important crop traits. To map genes and DNA sequences, it is necessary to have good genetic maps, which will allow the identification of quantitative trait loci (QTL). QTL mapping has contributed to the understanding of genetic architecture of complex plant traits, such as yield and fruit quality and disease resistance, that result from the cumulative effects of several genes (Fanizza et al., 2005; Fischer et al., 2004).

The development of genetic maps for auto-incompatible species, such as the yellow passion fruit $(2 n=18)$, is restricted due to the unfeasibility of obtaining traditional mapping populations based on inbred lines, like $\mathrm{F}_{2}, \mathrm{BC}_{1}$, or RILs. For this reason, yellow passion fruit linkage maps were generally constructed using a strategy known as two-way pseudo-testcross (Grattapaglia and Sederoff, 1994), based on monoparental dominant markers segregating in a 1:1 fashion. Due to the lack 
of information from these markers in one of the parents, two individual maps are constructed (i.e., one for each parental genotype). We have previously obtained maps based on random amplified polymorphic DNA (RAPD) and AFLP markers (Carneiro et al., 2002; Lopes et al., 2006) for the parents of a full-sib family derived from a cross between the Brazilian accessions IAPAR-123 and IAPAR-06. The homology of these linkage groups (LGs) was established using AFLP alleles that were present in both parents and hence segregate in a 3:1 ratio (Moraes, 2005). These biparental loci were placed as accessory markers on the individual maps.

Biparental markers that segregate in 3:1 (dominant), 1:2:1 (codominant), and 1:1:1:1 (codominant) ratios can be used to integrate individual linkage maps, as was recently done for sugarcane (Saccharum L. spp.) (Garcia et al., 2006). Dominant markers provide less information in linkage analyses than do codominants, and the loci that segregate in a $1: 1: 1: 1$ or $1: 2: 1$ fashion are highly informative (Wu et al., 2002).

Different numbers of alleles can segregate at each $\mathrm{F}_{1}$ locus, and different loci configurations should occur. These features cause difficulties in detection of the recombination events and consequently in construction of integrated maps, as the linkage phases at every pair of loci are not known (Maliepaard et al., 1997, 1998; Wu et al., 2002). In the first studies on linkage in outcrossing species (Arus et al., 1994; Ritter et al., 1990; Ritter and Salamini, 1996), the parental linkage phases and recombination frequencies were estimated separately. Later, Maliepaard et al. (1997) proposed a statistical approach for the estimation of recombination frequencies using loci with a variable number of alleles segregating in full-sib families. The authors also presented the LOD score formula for several types of configurations at every pair of markers. Maliepaard's approach was implemented in the software package JoinMap V3 (van Ooijen and Voorrips, 2001). More recently, Wu et al. (2002) proposed an alternative strategy that applies maximumlikelihood methods for simultaneous estimation of linkage and linkage phases, resolving several of the difficulties pointed out by Maliepaard et al. (1997). This method was successfully used in a sugarcane population (Garcia et al., 2006).

This study deals with construction of an integrated linkage map in the yellow passion fruit using the same full-sib progeny used to construct the RAPD- and AFLP-based maps (Carneiro et al., 2002; Lopes et al., 2006), applying a new method for incorporating information from markers segregating in different ratios as proposed by $\mathrm{Wu}$ et al. (2002). We combined dominant and codominant markers, particularly microsatellite loci. To our knowledge, this is the first integrated genetic map of a passion fruit species.

\section{Materials and Methods}

Plant materials, extraction, and Quantification of DNA. Carneiro et al. (2002) obtained the mapping population used in this study. In short, it derives from a cross between two non-inbred clones: IAPAR-06 (male parent), which was introduced from Morocco, and IAPAR-123 (female parent), which is a selection from the Brazilian 'Maguary' commercial population. Both clones belong to the Passiflora collection of the Instituto Agronômico do Paraná (IAPAR, Londrina, Brazil). This mating produced the $160 \mathrm{~F}_{1}$ individuals used in the present study, which were kept in a greenhouse and propagated by cuttings. Genomic DNA was extracted from $300 \mathrm{mg}$ of lyophilized leaf tissues using the cetyl trimethyl ammonium bromide (CTAB) method as described by Murray and Thompson (1980). DNA concentrations were estimated by electrophoresis on ethidium bromide-stained agarose gels using appropriate molecular weight standards.

AFLP DATA. The protocol for generating the AFLP data was described in detail by Lopes et al. (2006). A total of 253 monoparental markers that segregated in the population in a $1: 1$ ratio were used, as were 116 biparental loci segregating in a 3:1 ratio.

Microsatellite MARKers. One hundred seven microsatellite primers developed from enriched genomic libraries (Oliveira et al., 2005) were tested. All primer sequences are available upon consultation with the authors. Amplification reactions were done in a $20-\mu \mathrm{L}$ final volume using $20 \mathrm{ng}$ of DNA and four different mixes according to each locus (Table 1). Mix 1 contained $20 \mathrm{ng}$ of DNA, PCR buffer $1 \times, 1.5 \mathrm{~mm}$ $\mathrm{MgCl}_{2}, 200 \mu \mathrm{M}$ of each dNTP, $0.3 \mu \mathrm{M}$ of each forward and reverse primer, and $0.5 \mathrm{U}$ of Taq DNA polymerase (Fermentas, Inc., Hanover, MD). The buffer concentration was doubled in Mix 2. Mix 3 and Mix 4 both had $2.5 \mathrm{~mm} \mathrm{MgCl}_{2}$, and Mix 4 had a dNTP concentration of $350 \mu \mathrm{M}$.

A two-touchdown amplification program was designed. A denaturation step at $94{ }^{\circ} \mathrm{C}$ for 5 min initiated the PCR. Then, eight cycles of $40 \mathrm{~s}$ at $94{ }^{\circ} \mathrm{C}, 40 \mathrm{~s}$ at $60{ }^{\circ} \mathrm{C}$ with a $0.5^{\circ} \mathrm{C}$ decrease per cycle, and $50 \mathrm{~s}$ at $72{ }^{\circ} \mathrm{C}$ were carried out. An additional 24 cycles at an annealing temperature of $56^{\circ} \mathrm{C}$ and a final extension at $72{ }^{\circ} \mathrm{C}$ for $5 \mathrm{~min}$ completed this program (TD60). A second PCR program was also carried out (TD56): denaturation was induced at $94{ }^{\circ} \mathrm{C}$ for $5 \mathrm{~min}$, and annealing was performed at $56^{\circ} \mathrm{C}$ for $40 \mathrm{~s}$ with a $0.5{ }^{\circ} \mathrm{C}$ decrease per cycle for 12 cycles. An additional 20 cycles at an annealing temperature of $50^{\circ} \mathrm{C}$ with a final extension at $72{ }^{\circ} \mathrm{C}$ for 5 min completed the amplification process. For some of the primer pairs, specific annealing temperatures at 60,56 , or $52{ }^{\circ} \mathrm{C}$ were adopted. Amplifications were conducted on a GeneAmp PCR System 9700 thermocycler (Applied Biosystems, Foster City, CA). The amplification products were mixed in a 2:1 proportion with a denaturing loading buffer; afterward, samples were loaded onto $6 \%$ polyacrylamide$7 \mathrm{M}$ urea gels and electrophoresed in $1 \times$ TBE buffer at $80 \mathrm{~W}$ for 2 h. Silver staining was performed according to Creste et al. (2001).

Notation. AFLP loci nomenclature consisted of two letters and five or six numbers. The letters represent the enzymes used in the digesting reaction $(E c o \mathrm{RI}=\mathrm{E}, M s e \mathrm{I}=\mathrm{M}$, and $P s t \mathrm{I}=\mathrm{P})$, and the first two numbers indicate the combination of primers with their arbitrary nucleotides used in the selective amplification, as described by Lopes et al. (2006). The last three or four numbers correspond to the molecular size (in bp) of the AFLP fragment. For microsatellite loci, the code $P E$ (from $P$. edulis) was adopted followed by the locus number. When more than one locus was amplified by the same primer pair, the size of the polymorphic allele was added to the code.

Wu's notation about loci-segregation patterns precedes all the marker codes [e.g., D1-EM19246, D1-PE07 (see Fig. 1)]. In brief, we assumed the presence of a maximum number of four codominant alleles, denoted by $a, b, c$, or $d$. All of these are dominant over 0 (or null) alleles. " $\mathrm{D}_{1}$ " markers (denoted $a 0 \times$ $00)$ are heterozygous for their presence in one parent (IAPAR123) and homozygous for their absence in the other (IAPAR06), while" $\mathrm{D}_{2}$ " loci have the inverted genetic configuration $(00 \times a 0)$. "C" loci are heterozygous in both parents and segregate in a $3: 1$ fashion $(a 0 \times a 0)$, whereas " $\mathrm{B}_{3}$ " loci are heterozygous in both parents and segregate 1:2:1 (e.g., $a b \times a b)$. 


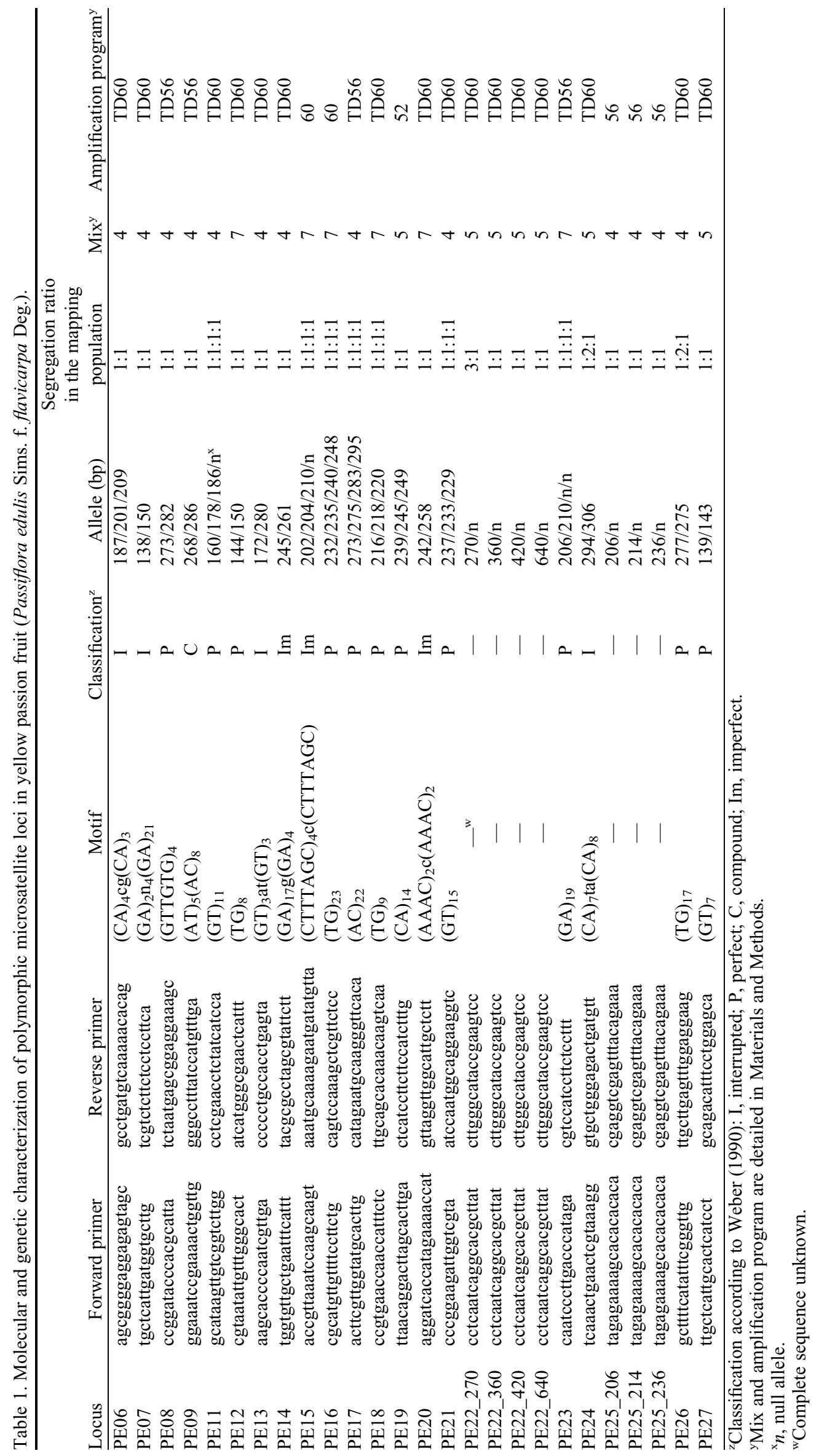


LG-I

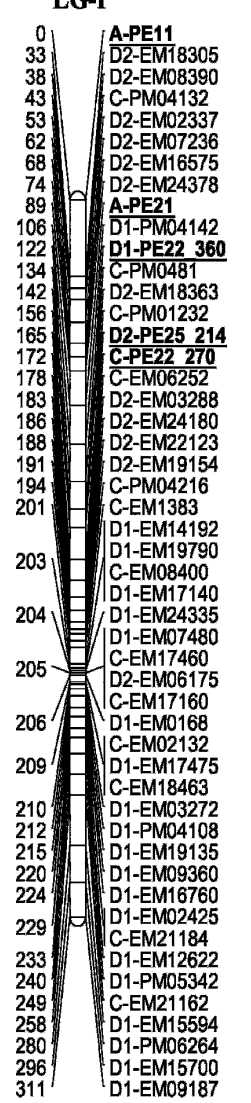

LG-V

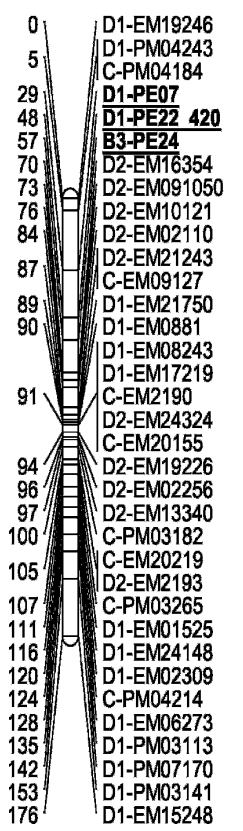

LG-II

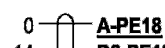

14 - D2-PE19

31 D2-EM21161

92 1 / D2-EM01236

96 1 D2-EM20172

111 . C-EM1992

123 HC-EM16725

126 基 C-EM15800

129 倠 D1-EM02230

131 D D1-EM151050

134 $=$ D1-EM24235

140 14 D1-EMO4192

143 D1-EM20131

144 HD1-EM20231

D1-EM22112

160 D1-EM2296

199 $\leadsto$ D1-EM16308

234 A-PE15
LG-III

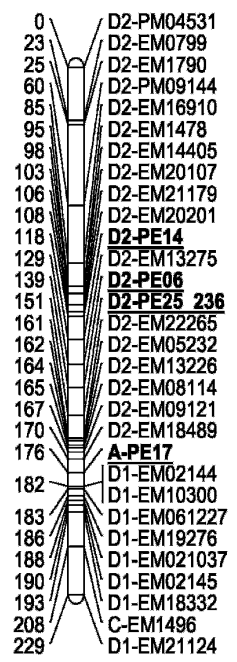

LG-IV (IAPAR 06)

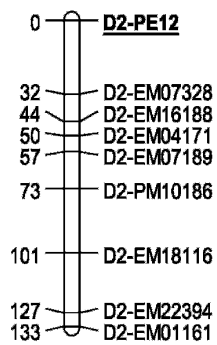

LG-IV (IAPAR 123)

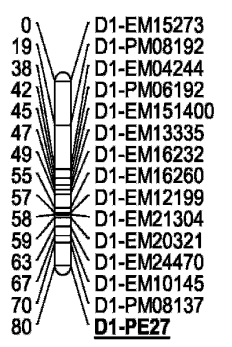

the problems caused by the realization of multiple tests taken into account using the false-discovery rate (Storey and Tibshirani, 2003). All linkage analyses were done using OneMap software (Margarido et al., 2007) as described by Garcia et al. (2006), using the methods proposed by Wu et al. (2002). First, cosegregation groups were established using LOD score $\geq 5.0$ and recombination fraction $\leq 0.35$ with simultaneous estimation of linkage phases based on posterior probabilities and using the EM algorithm (Dempster et al., 1977). The linkage phases between uninformative combinations of markers (" $\mathrm{D}_{1}$ " vs. " $\mathrm{D}_{2}$ ") were estimated indirectly when possible. Second, the order of markers within cosegregation groups was estimated using the rapid chain delineation (Doerge, 1996) combined with the ripple algorithm (Lander et al., 1987) using: 1) windows with six markers and based on the sum of adjacent recombination fraction (SARF); 2) windows with three markers and based on the likelihood of each suborder (Lu et al., 2004). Finally, three-point estimates of recombination fractions were calculated and converted into linkage distances using the Kosambi map function (Kosambi, 1944).

\section{Results}

The ratio of polymorphism found at those AFLP loci was $13.0 \%$, which corresponds to half of the proportion found at the microsatellite loci (24.7\%). From the 253 AFLP monoparental markers, 114 and 139 of them were heterozygous for their presence in the male (IAPAR-06) and female (IAPAR-123) parent, respectively, and homozygous for their absence in the other. In addition, 116 AFLP biparental loci, segregating in a $3: 1$ ratio, were used for constructing the integrated genetic map.

We tested 107 microsatellite primer pairs, from which 85 pro-

Fig. 1. Integrated genetic map of the yellow passion fruit based on AFLP and microsatellite markers (bold and underlined); $\mathrm{LG}=$ linkage group.

"A" loci are those that segregated in a 1:1:1:1 ratio, involving two alleles and two null alleles $(a 0 \times b 0)$; three alleles and one null allele $(a b \times c 0)$; or three $(a b \times a c)$ or four non-null alleles $(a b \times c d)$.

LINKAGE AND MARKER ORDER. Initially, statistical tests for the identification of segregation distortion were carried out, with duced patterns with good reproducibility. Twenty-one primer pairs revealed a polymorphism between the parents (Table 1). Most of the PCR fragments were of the same length, as was expected from sequence analysis, but at two loci, PE22 and $P E 25$, the size of the amplicons was different from those expected, consistent with the primer design (241 and $246 \mathrm{bp}$, 
respectively). Actually, these primer pairs revealed seven loci: PE22_270, PE22_360, PE22_420, PE22_640, PE25_206, PE25_214, and PE25_236. Because they segregated in a Mendelian fashion, they were used for the map construction. All were placed on the LG, with the exception of PE22_640.

Of the 26 microsatellite loci, $16(61.5 \%)$ segregated in a $1: 1$ fashion. Ten were heterozygous in IAPAR-06 and six in IAPAR-123, but null in the other parent. The remaining 10 loci $(38.5 \%)$ were heterozygous in both parents: one segregated in a $3: 1$ proportion, two in a 1:2:1 fashion, and seven loci were highly informative as they segregated at $1: 1: 1: 1$.

The configuration $00 \times a 0\left(\right.$ " $\mathrm{D}_{2}$ ") was noted at PE22_640, PE25_206, and PE25_214 loci; IAPAR-06 was the parent that produced the amplicon. The reciprocal configuration (" $\mathrm{D}_{1}$ ") was observed at PE22_360 and PE22_420. Just one allele was detected at PE22_270 locus $(a 0 \times a 0)$ that segregated at 3:1 (dominant marker), as genotypes $a a$ and $a 0$ could not be distinguished by their molecular phenotypes (" $\mathrm{C}$ " configuration).

The following loci segregated at 1:1:1:1 ("A"; i.e., all possible genotypes were distinguished based on their molecular profiles): in PE11 and PE15 $(a b \times c 0)$ loci, the null allele was present in IAPAR-06; the $a 0 \times b 0$ configuration characterized the PE23 locus; PE16 and PE17 showed four alleles $(a b \times c d)$, while three alleles were identified at PE18 and PE21 loci $(a b \times$ $a c)$. Otherwise, the configuration $a b \times a b$ (" $\mathrm{B}_{3}$ ") characterized PE24 and PE26 loci that segregated at 1:2:1.

The female parent was informative at PE07, PE09, PE20, and $P E 27(a b \times a a)$, which segregated at $1: 1$ in the population (" $\mathrm{D}_{1}$ "), while the male parent was informative at $P E 08$, PE12, PE13, PE14, and PE25_236 $(a a \times a b)$ and at PE06 and $P E 19$ loci $(c c \times a b)$, which also segregated in a $1: 1$ proportion (" $\mathrm{D}_{2}$ ").

The total number of markers with nondistorted segregation assembled on the map was 308, 126 of them coming from IAPAR-123 (120 AFLP and 6 SSR markers) and 108 from IAPAR-06 (98 AFLP and 10 SSR markers); 74 were biparental loci, 64 being AFLPs and 10 SSRs. By using the method of Wu et al. (2002), 296 markers (96\%) were assembled into nine LGs. However, one of these LGs comprised 107 markers. By removing a specific AFLP marker, to which there were a few markers linked, the LG split into two new ones (Fig. 1).

The haploid chromosome number of $P$. edulis $\mathrm{f}$. flavicarpa is $n=9$ (Cuco et al., 2005), but here we found 10 LG (Fig. 1). Due to the lack of biparental markers, two other LGs remained as individuals, which were denoted LG-IV (06) and LG-IV (123). By exclusion, we speculate that they are homologous.

Taking into account the AFLP markers, 82.1\% (179/218) and $68.8 \%(44 / 64)$ of the loci that segregated at $1: 1$ and $3: 1$, respectively, were assembled on the LG. All microsatellite loci that segregated at $3: 1$ and $1: 2: 1$ were placed on the map, as were 12 out of 16 microsatellite markers that segregated at a 1:1 proportion. Out of seven that segregated in a $1: 1: 1: 1$ fashion, only two were not placed on the integrated map.

The genetic linkage map comprised 243 markers $(78.9 \%$ of the total), and the LGs consisted of nine to 50 markers. On average, 24 markers were assigned per LG. The total map length was $1687 \mathrm{cM}$, with a marker density of $6.9 \mathrm{cM}$. The greater distance was $35 \mathrm{cM}$ (LG-II and LG-III). Due to the approach here used for ordering, no markers were included as accessories on the integrated map, in contrast to the first maps published (Carneiro et al., 2002; Lopes et al., 2006).

\section{Discussion}

As mentioned above, $\approx 25 \%$ of the microsatellite loci were polymorphic; this percentage includes the primers that revealed more than one Mendelian locus. Microsatellite markers amplifying more than one set of fragments were previously reported in apple (Malus domestica Borkh.) (Kenis and Keulemans, 2005), kiwifruit (Actinidia chinensis Planch.) (Testolin et al., 2001), and Lens L. (Hamwich et al., 2005). The ratio of polymorphism was low in the yellow passion fruit, considering the information reported for other cultivated tropical species, such as cocoa (Theobroma cacao L.) (52\%; Pugh et al., 2004) and rubber tree (Hevea Aubl.) (46\%; Lespinasse et al., 2000). The size of these genomes and the relatively small number of loci evaluated here (85), or even the genetic distance between the parents used for obtaining the mapping population, may be responsible for the discrepancy. The same was observed at the passion fruit AFLP loci, compared with other highly heterozygous species with a strong self-incompatible mechanism that ensures outcrossing for reproduction. The apple, for example, was reported to have $28.5 \%$ at AFLP loci (Kenis and Keulemans, 2005). Low levels of molecular polymorphisms were shown in $P$. edulis and P. edulis f. flavicarpa using RAPD (Fajardo et al., 1998) and chloroplast DNA analyses (Sanchez et al., 1999).

In both genetic maps previously constructed in our laboratory using the same $F_{1}$ population, the RAPD and AFLP markers were distributed in nine LGs. They were developed applying the two-way pseudo-testcross mapping strategy and markers that segregated in a 1:1 ratio. In addition, Moraes (2005) developed a new AFLP-based genetic map using markers that segregated in ratios of $1: 1$ and $3: 1$. For this purpose, computational software programs TreeMap (Coelho, 2005) and MapMaker/EXP (Lander et al., 1987) were used. Due to the presence of common biparental markers $(3: 1)$, the homology between the individual LGs was established. The parental linkage maps were composed of nine LGs (Table 2). The 3:1 loci were placed as accessory markers on the maps. Conversely, in the present study we placed these markers as framework loci in the integrated map.

For most of the LG of the integrated genetic map, the marker orders were consistent with those of the individual maps. No substantial changes were observed. The integrated map combines loci that segregate in one or both parents. Considering a locus that is heterozygous in both parents, the estimated recombination fraction corresponds to the average between the recombination frequencies that occurred in the parental meiosis. This combined estimate should slightly alter the marker orders on the integrated linkage map (Maliepaard et al., 1998) in comparison with the individual maps. Moreover, statistical problems may provoke inverted marker orders, as pointed out to explain the differences found in Populus L. maps (Yin et al., 2002). The effects of population size, marker spacing, ratio of dominant to codominant markers, and missing values also contributed to errors in the estimation of marker orders (Brondani et al., 2002; Hackett et al., 2003). In the present study, the inversions probably occurred due to the inclusion of biparental markers (1:1 and 3:1). The map became more saturated and LGs longer, diminishing the linkage distances and allowing some inversions in the marker orders. 
Table 2. Comparison between the parental linkage maps constructed using the two-way pseudo-testcross approach (data not shown) and the integrated map of yellow passion fruit (Passiflora edulis Sims. f. flavicarpa Deg.).

\begin{tabular}{|c|c|c|c|c|}
\hline Attribute & & Female parent (IAPAR-123) & Male parent (IAPAR-06) & Integrated map \\
\hline \multirow[t]{2}{*}{ Markers assigned in the framework (no.) } & $1: 1$ & 57 & 63 & 191 \\
\hline & $1: 2: 1$ plus $1: 1: 1: 1$ & $-^{\mathrm{z}}$ & $-^{\mathrm{z}}$ & 7 \\
\hline Accessory markers (no.) & $3: 1^{\mathrm{y}}$ & 60 & 58 & 0 \\
\hline Loci (total no.) & & 179 & 153 & $243^{\mathrm{x}}$ \\
\hline Linkage groups (no.) & & 9 & 9 & 10 \\
\hline Length of the larger group $(\mathrm{cM})$ & & 94 & 212 & 311 \\
\hline Length of the smaller group (cM) & & 11 & 11 & 80 \\
\hline Average length of the groups (cM) & & 52.4 & 80.4 & 168.7 \\
\hline Average distance of the markers (cM) & & 8.3 & 11.5 & 6.9 \\
\hline
\end{tabular}

${ }^{\mathrm{z}}$ Not used.

${ }^{\mathrm{y}}$ Markers used for establishment of the homology between the LGs generated by the two-way pseudo-testcross.

×55 markers were not placed on the framework.

wAccording to the Kosambi map function.

We have stated that AFLP clusters occurred in the yellow passion fruit maps (Lopes et al., 2006; Moraes, 2005); EcoRI/ $M$ seI digestions produce fragments that occur in clusters along the chromosomes (Alonso-Blanco et al., 1998; Keim et al., 1997), while the PstI/MseI combinations reveal markers that are distributed randomly in the genome (Vuylsteke et al., 1999). This may also contribute to incorrect marker orders.

Although biparental markers were available, some authors preferred to develop individual maps, as one definitive linkage map for each cultivar is desired (Kenis and Keulemans, 2005; Testolin et al., 2001). Others preferred to construct integrated maps using the JoinMap software (Hamwich et al., 2005; Risterucci et al., 2000). Recently, Garcia et al. (2006) published a new sugarcane linkage map using Wu's approach; according to the authors, the simultaneous maximum-likelihood estimation of linkage and linkage phases was more efficient, allowing detection of linkage for a higher number of markers that were assigned to 131 cosegregation groups, in comparison with the previous 98 obtained with JoinMap V3.

The integrated map described herein combines AFLP and microsatellite markers (Table 2; Fig. 1). About $69 \%$ of the 3:1segregating markers were placed into eight LGs, with an average of 5.5 markers per group. Similar results in terms of distribution of the 3:1 markers were reported in grape using Vitis rupestris Scheele and Vitis arizonica Engelm. (Doucleff et al., 2004). Although the number of microsatellite markers was relatively small (26), integration of the individual passion fruit maps was possible with no substantial deviation of the linear marker orders. In the previous maps based on the two-way testcross strategy, only part of the genetic information from the parents was used (e.g., the 1:1-segregating loci). The construction of an integrated map will allow us to compare and group the information from other mapping populations in the future. In addition, such a map represents a valuable source of probes which can be used to assign the LGs to P. edulis chromosomes.

Mapping quantitative traits is crucial for all agronomic species. Our research group has already studied several quantitative traits related to yield and fruit quality in the same $F_{1}$ population (IAPAR-06 vs. IAPAR-123) (Moraes et al., 2005) as well as mapped an important quantitative resistance locus (QRL) related to the response to Xanthomonas axonopodis pv. passiflorae. This pathogen is frequently found in passion fruit orchards and is very destructive for the crop. The QRL was identified between the markers EM01156 and EM1 1800 (herein placed into LG-VIII; Fig. 1) and explained $16.5 \%$ of the total phenotypic variation for diseased leaf area in the segregating population (Lopes et al., 2006). However, several of the resistance loci are multiallelic, and this appears to be the mechanism of plant response to $X$. axonopodis infection. Lin et al. (2003) presented a statistical model for mapping QTL in outcrossing species, dealing with the problems that occur in this situation. They discussed the possibility that the failure to characterize a correct linkage phase could lead to bias in the estimation of QTL position and effects and presented a method for correcting the problem. Their technique, however, is based on a previous genetic map built using the method of Wu et al. (2002), which was used in our research. Therefore, we believe that integrated maps with good estimation of linkage phases provide a sound basis for QTL mapping and will provide the correct manipulation of favorable QRL alleles.

\section{Literature Cited}

Alonso-Blanco, C., A.J.M. Peeters, M. Koornneef, C. Lister, C. Dean, N. van den Bosh, J. Pot, and M.T.R. Kuiper. 1998. Development of an AFLP based linkage map of Ler Col and Cvi Arabidopsis thaliana ecotypes and construction of a Ler/Cvi recombinant inbred line population. Plant J. 14:259-271.

Arus, P., C. Olarte, M. Romero, and F. Vargas. 1994. Linkage analysis of 10 isozyme genes in $F_{1}$ segregating almond progenies. J. Amer. Soc. Hort. Sci. 119:339-344.

Brondani, R.P.V., C. Brondani, and D. Grattapaglia. 2002. Towards a genus-wide reference linkage map for Eucalyptus based exclusively on highly informative microsatellite markers. Mol. Genet. Genomics 267:338-347.

Carneiro, M.S., L.E.A. Camargo, A.S.G. Coelho, R. Vencovsky, R.P. Leite, Jr., N.M.C. Stenzel, and M.L.C. Vieira. 2002. RAPD-based genetic linkage maps of yellow passion fruit (Passiflora edulis Sims f. flavicarpa Deg.). Genome 45:670-678.

Coelho, A.S.G. 2005. TreeMap: software para construção de mapas genéticos em cruzamentos derivados de genótipos não-endogâmicos. Univ. Federal de Goiânia, Goiânia, Brazil.

Creste, S., A. Tulmann-Neto, and A. Figueira. 2001. Detection of single sequence repeat polymorphisms in denaturing polyacrylamide sequencing gels by silver staining. Plant Mol. Biol. Rpt. 19:299-306. 
Cuco, S.M., M.L.C. Vieira, M. Mondin, and M.L.R. Aguiar-Perecin. 2005. Comparative karyotype analysis of three Passiflora L. species and cytogenetic characterization of somatic hybrids. Caryologia 58:220-228.

Dempster, A.P., N.M. Laird, and D.B. Rubin. 1977. Maximum likelihood from incomplete data via the EM algorithm. J. Royal Stat. Soc. Ser. B 39:1-38.

Doerge, R.W. 1996. Constructing genetic maps by rapid chain delineation. J. Agr. Genomics 2:6.

Doucleff, M., Y. Jin, F. Gao, S. Riaz, A.F. Krivanek, and M.A. Walker. 2004. A genetic linkage map of grape utilizing Vitis rupestris $\times$ Vitis arizonica. Theor. Appl. Genet. 109:1178-1187.

Fajardo, D., F. Angel, M. Grum, J. Tohme, M. Lobo, W.M. Roca, and I. Sanchez. 1998. Genetic variation analysis of the genus Passiflora L. Euphytica 101:341-347.

Fanizza, G., F. Lamaj, L. Costantini, R. Chaabane, and M.S. Grando. 2005. QTL analysis for fruit yield components in table grapes (Vitis vinifera). Theor. Appl. Genet. 111:658-664.

Fischer, B.M., I. Salakhutdinov, M. Akkurt, R. Eibach, K.J. Edwards, R. Töpfer, and E.M. Zyprian. 2004. Quantitative trait locus analysis of fungal disease resistance factors on a molecular map of grapevine. Theor. Appl. Genet. 108:501-515.

Garcia, A.A.F., E.A. Kido, A.N. Meza, H.M.B. Souza, L.R. Pinto, M.M. Pastina, C.S. Leite, J.A.G. da Silva, E.C. Ulian, A. Figueira, and A.P. Souza. 2006. Development of an integrated genetic map of a sugarcane (Saccharum spp.) commercial cross based on a maximumlikelihood approach for estimation of linkage and linkage phases. Theor. Appl. Genet. 112:298-314.

Grattapaglia, D. and R. Sederoff. 1994. Genetic linkage maps of Eucalyptus grandis and Eucalyptus urophylla using a pseudotestcross: mapping strategy and RAPD markers. Genetics 137: 1121-1137.

Hackett, C.A., B. Pande, and G.C. Bryan. 2003. Constructing linkage maps in autotetraploid species using simulated annealing. Theor. Appl. Genet. 106:1107-1115.

Hamwich, A., S.M. Udupa, W. Choumane, A. Sarker, F. Dreyer, C. Jung, and M. Baum. 2005. A genetic linkage map of Lens sp. based on microsatellite and AFLP markers and the localization of Fusarium vascular wilt resistance. Theor. Appl. Genet. 110:669-677.

Keim, P., J.M. Schupp, S.E. Travis, K. Clayton, T. Zhu, L. Shi, A. Ferreira, and D.M. Webb. 1997. A high-density soybean genetic map based on AFLP markers. Crop Sci. 37:537-543.

Kenis, K. and J. Keulemans. 2005. Genetic linkage maps of two apple cultivars (Malus domestica Borkh.) based on AFLP and microsatellite markers. Mol. Breed. 15:205-219.

Kosambi, D.D. 1944. The estimation of map distance from recombination values. Ann. Eugen. 12:172-175.

Lander, E.S., P. Green, J. Abrahamson, A. Barlow, M.J. Daly, E.E. Lincoln, and L. Newburg. 1987. MapMaker: an interactive computer package for constructing primary genetic linkage maps of experimental and natural populations. Genomics 1:174-181.

Lespinasse, D., G.M. Rodier, L. Grivet, A. Leconte, H. Legnate, and M. Seguin. 2000. A saturated genetic linkage map of rubber tree (Hevea spp.) based on RFLP, AFLP, microsatellite and isozymes markers. Theor. Appl. Genet. 100:127-138.

Lin, M., X.Y. Lou, M. Chang, and R. Wu. 2003. A general statistical framework for mapping quantitative trait loci in nonmodel systems: issue for characterizing linkage phases. Genetics 165:901-913.

Lopes, R., M.T.G. Lopes, M.S. Carneiro, F.P. Matta, L.E.A. Camargo, and M.L.C. Vieira. 2006. AFLP linkage analysis and mapping of resistance genes to Xanthomonas axonopodis pv. passiflorae in yellow passion fruit. Genome 49:17-29.

Lu, Q., Y. Cui, and R. Wu. 2004. A multilocus likelihood approach to joint modeling of linkage, parental diplotype and gene order in a fullsib family. BMC Genet. 5:1-14.

Maliepaard, C., F.H. Alston, G. van Arkel, L.M. Brown, E. Chevreau, F. Dunemann, K.M. Evans, S. Gardiner, P. Guilford, A.W. van Heusden, J. Janse, F. Laurens, J.R. Lynn, A.G. Manganaris, A.P.M.
Den Nijs, N. Periam, E. Rikkerink, P. Roche, C. Ryder, S. Sansavini, H. Schmidt, S. Tartarini, J.J. Verhaegh, M. Vrielink-van Ginkel, and G.J. King. 1998. Aligning male and female linkage maps of apple (Malus pumila Mill.) using multi-allelic markers. Theor. Appl. Genet. 97:60-73.

Maliepaard, C., J. Jansen, and J.W. van Ooijen. 1997. Linkage analysis in a full-sib family of an outbreeding plant species: overview and consequences for applications. Genet. Res. 70:237-250.

Margarido, G.R.A., A.P. Souza, and A.A.F. Garcia. 2007. OneMap: software for genetic mapping in outcrossing species. Hereditas 144: 78-79.

Moraes, M.C. 2005. Mapas de ligação e mapeamento de QTL ("quantitative trait loci") em maracujá-amarelo (Passiflora edulis Sims f. flavicarpa Deg.). Univ. of São Paulo, Escola Superior de Agricultura "Luiz de Queiroz", Piracicaba, Brazil. PhD Diss.

Moraes, M.C., I.O. Geraldi, F.P. Matta, and M.L.C. Vieira. 2005. Genetic and phenotypic parameter estimates for yield and fruit quality traits from a single wide cross in yellow passion fruit. HortScience 40:1978-1981.

Murray, M.G. and W.F. Thompson. 1980. Rapid isolation of high molecular weight plant DNA. Nucleic Acids Res. 8:4321-4325. Oliveira, E.J., J.G. Padua, M.I. Zucchi, L.E.A. Camargo, M.H.P. Fungaro, and M.L.C. Vieira. 2005. Development and characterization of microsatellite markers from the yellow passion fruit (Passiflora edulis f. flavicarpa). Mol. Ecol. Notes 5:331-333.

Pugh, T., O. Fouet, A.M. Risterucci, P. Brottier, M. Abouladze, C. Deletrez, B. Courtois, D. Clement, P. Larmande, J.A.K. N'goran, and C. Lanaud. 2004. A new cacao linkage map based on codominant markers: development and integration of 201 new microsatellite markers. Theor. Appl. Genet. 108:1151-1161.

Risterucci, A.M., L. Grivet, J.A.K. N'goran, I. Pieretti, M.H. Flament, and C. Lanaud. 2000. A high-density linkage map of Theobroma cacao L. Theor. Appl. Genet. 101:948-955.

Ritter, E., C. Gebhardt, and F. Salamini. 1990. Estimation of recombination frequencies and construction of RFLP linkage maps in plants from crosses between heterozygous parents. Genetics 125:645-654.

Ritter, E. and F. Salamini. 1996. The calculation of recombination frequencies in crosses of allogamous plant species with applications to linkage mapping. Genet. Res. 67:55-65.

Sanchez, I., F. Angel, M. Grum, M.C. Duque, M. Lobo, J. Tohme, and W. Roca. 1999. Variability of chloroplast DNA in the genus Passiflora L. Euphytica 106:15-26.

Storey, J.D. and R. Tibshirani. 2003. Statistical significance for genome-wide studies. Proc. Natl. Acad. Sci. USA 5:9440-9445.

Testolin, R., W.G. Huang, O. Lain, R. Messina, A. Vecchione, and G. Cipriani. 2001. A kiwifruit (Actinidia spp.) linkage map based on microsatellites and integrated with AFLP markers. Theor. Appl. Genet. 103:30-36.

van Ooijen, J.W. and R.E. Voorrips. 2001. JoinMap, version 3.0, software for the calculation of genetics linkage maps. Plant Research International, Wageningen, The Netherlands.

Vieira, M.L.C. and M.S. Carneiro. 2004. Passiflora spp. passionfruit, p. 435-453. In: R.E. Litz (ed.). Biotechnology of fruit and nut crops. CABI Publishing, Oxford, U.K.

Vuylsteke, M., R. Mank, R. Antonise, E. Bastiaans, M.L. Senior, C.W. Stuber, A.E. Melchinger, T. Lubberstedt, X.C. Xia, P. Stam, M. Zabeau, and M. Kuiper. 1999. Two high-density AFLP linkage maps of Zea mays L.: analysis of distribution of AFLP markers. Theor. Appl. Genet. 99:921-935.

Weber, J.L. 1990. Informativeness of human $(\mathrm{dC}-\mathrm{dA})_{n} \cdot(\mathrm{dG}-\mathrm{dT})_{n}$ polymorphisms. Genomics 7:524-530.

Wu, R., C.X. Ma, I. Painter, and Z.-B. Zeng. 2002. Simultaneous maximum likelihood estimation of linkage and linkage phases in outcrossing species. Theor. Popul. Biol. 61:349-363.

Yin, T., Z. Zhang, M. Huang, M. Wang, Q. Zhuge, S. Tu, L.H. Zhu, and R. Wu. 2002. Molecular linkage maps of the Populus genome. Genome 45:541-555. 